\title{
Breve história da sistemática de Agaricaceae (Fungi) e
} distribuição no Brasil

\author{
Felipe Wartchow
}

Universidade Federal da Paraíba, Centro de Ciências Exatas e da Natureza, Departamento de Sistemática e Ecologia, Programa de Pós-Graduação em Ciências Biológicas, Cidade Universitária, João Pessoa 58051-900, Paraíba, Brasil. E-mail: fwartchow@yahoo.com.br

Universidade Federal da Paraíba, Centro de Ciências Aplicadas e Educação, Programa de Pós-Graduação em Ecologia e Monitoramento Ambiental, Campus IV - Litoral Norte, Rio Tinto 58280-000, Paraíba, Brasil.

Universidade Federal do Rio Grande do Norte, Centro de Biociências, Programa de Pós-Graduação em Sistemática e Evolução, Campus Universitário, Lagoa Nova, Natal 59078-970, Rio Grande do Norte, Brasil.

Universidade Federal de Pernambuco, Centro de Ciências Biológicas, Programa de Pós-Graduação em Biologia de Fungos, Av. Nelson Chaves, Cidade Universitária, Recife 50373970, Pernambuco, Brasil.

Wartchow F. (2018) Breve história da sistemática de Agaricaceae (Fungi) e distribuição no Brasil. Pesquisa e Ensino em Ciências Exatas e da Natureza, 2(2): 130-147. http://dx.doi.org/10.29215/pecen.v2i2.1067

Editora acadêmica: Lydia Dayanne Maia Pantoja. Recebido: 24 Maio 2018. Aceito: 23 Setembro 2018. Publicado: 28 Dezembro 2018.

Resumo: A família Agaricaceae pertença à ordem Agaricales. No geral, as espécies agaricoides deste grupo são caracterizadas por possuir píleo squamuloso. Nesta revisão é apresentada uma breve história da sistemática desta família e distribuição das espécies agaricoides no Brasil desde meados do Século XIX até os dias atuais.

Palavras chave: Agaricales, Agaricomycetes, Basidiomycota, Neotrópico.

\section{Brief history of the systematic of Agaricaceae and the distribution in Brazil}

Abstract: The family Agaricaceae belongs to order Agaricales. In general the agaricoid species of this group are characterized by having squamulose pileus. This revision presents a brief history of the systematic of this group and distribution of their agaricoid species in Brazil since from early 19" Century to present days.

Key words: Agaricales, Agaricomycetes, Basidiomycota, Neotropic.

\section{Introdução}

A família Agaricaceae Fr. pertence à ordem Agaricales, classe Agaricomycetes do filo Basidiomycota. O conceito mais conhecido sobre sistemática do grupo foi proposto por Singer (1986), contando com 25 gêneros de basidioma agaricoide, a maioria ocorrendo em quase todos os continentes, distribuídos em quatro tribos (Leucocoprinae Singer, Agariceae Pat., Lepiotae Fayod e Cystodermatae Singer). Segundo Kirk et al. (2008), possui 85 gêneros e 1340 espécies.

Tradicionalmente, esta família compreendia fungos usualmente chamados de cogumelos ou chapéus-de-cobra, caracterizados por apresentarem basidiomas anuais, carnosos a membranáceos, sem zonações concêntricas, com píleo variando de $0.5 \mathrm{~cm}$ até aproximadamente $30 \mathrm{~cm}$. Em geral, apresentam escamas ou esquâmulas cobrindo parte de sua superfície, provenientes da ruptura de um véu ou anel, cujos restos ficam presos ao estipe ou são fugazes, 
esvaecendo logo cedo. As lamelas podem ser remotas, colariadas, livres a adnatas. O estipe é central, podendo raramente apresentar volva. A esporada varia do branco, creme ao rosado, e de marrom a quase preto. Os esporos são hialinos a méleos, com parede fina a espessa, com ou sem poro germinativo. A trama do himenóforo é regular a irregular, e as hifas do contexto podem apresentar ou não grampos-de-conexão. Os basídios são, em geral, clavados com quatro esterigmas. A superfície pilear varia de uma cútis pouco diferenciada a um epicutis do tipo himeniodermal, paliçádica, tricodermial ou com esferocistos ou dermatocistídios (Singer 1986; Pereira \& Putzke 1989).

O substrato varia, podendo ser humícola, lignícola e às vezes musícola (Pereira \& Putzke 1989), mas Macrolepiota stercoraria (Rick) Raithelh. foi descoberta crescendo sobre esterco (Raithelhuber 1987a).

Algumas espécies da família Agaricaceae são comestíveis (Singer 1986; Alexopoulos et al. 1996), como as do gênero Agaricus L.: Fr., por exemplo: Agaricus arvensis Schaeff., A. augustus Fr., $A$. campestris L. e $A$. rodmanii Peck. No Brasil, a principal espécie cultivada comercialmente é $A$. bisporus (J.E. Lange) Imbach, uma espécie exótica (Pereira \& Putzke 1989). Segundo Singer (1986), o gênero Macrolepiota Singer também possui várias espécies comestíveis. Outras espécies comestíveis pertencem a vários gêneros de Agaricaceae, como Leucoagaricus naucinus (Fr.) Singer e Chamaemyces fracidus (Fr.) Donk (Singer 1986). Chlorophyllum esculentum Massee também é comestível, apesar de Dennis (1952), Pegler \& Rayner (1969) e Singer (1986) considerarem sinônimo de $C$. molybdites (G. Mey.) Massee.

Algumas espécies tóxicas também são conhecidas, como Macrolepiota venenata Bon, várias espécies de Leucoagaricus e uma das espécies amarelas de Leucocoprinus (Singer 1986). Agaricus xanthodermus Genev., possui substâncias fenólicas tóxicas (Singer 1986), e provoca distúrbios gastrintestinais; Chlorophyllum molybdites (Figura 1) provoca náuseas, vômitos e diarréias (Meijer et al. 2007); as espécies do grupo Lepiota helveola provocam a mesma síndrome de Amanita phalloides (Singer 1986); na América do Sul, Lepiota locanensis Espinosa tem sido citada como tóxica (Singer 1986); e para o Japão, é relatado um caso de intoxicação nãoletal por L. neomastoidea Hongo, causando alugus distúrbios gastrointestinais como vômito e diarréia (Yokohama \& Yamaji 1981).

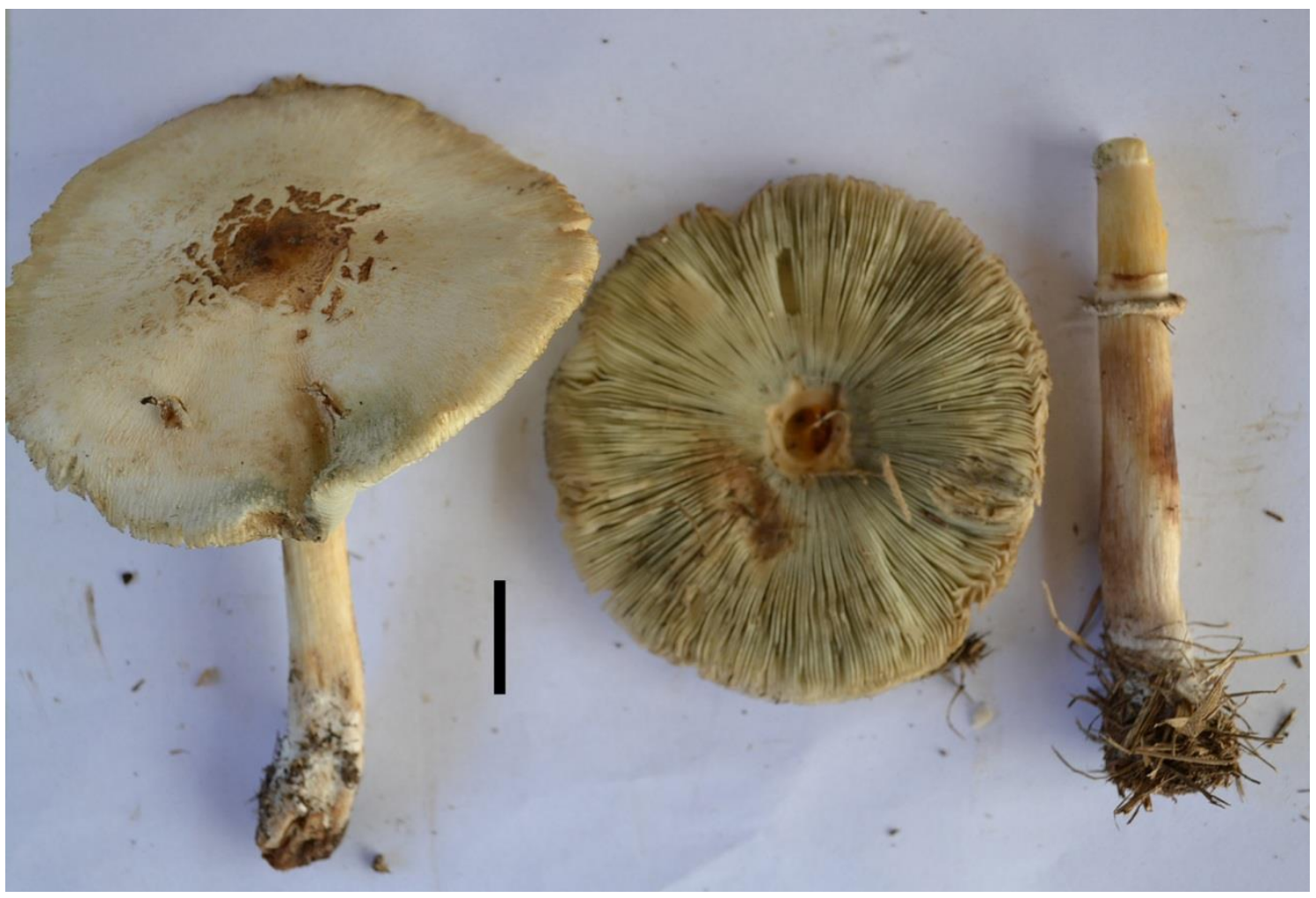

Figura 1. Chlorophyllum molybdites, espécie tóxica (Meijer et al. 2007). Foto: F. Wartchow. 
Existem também espécies de Agaricaceae vivendo em simbiose com formigas, como Leucoagaricus gongylophorus (Møller) Singer (Singer 1986; Fisher et al. 1994; Spielmann \& Putzke 1998) e Leucoagaricus weberi J.J Muchovej, Della Lucia \& R.M.C. Muchovej (Muchovej et al. 1991). Leucocoprinus gongylophorus (A. Møller) R. Heim foi encontrado em um ninho ativo de formiga por Bononi et al. (1981a), mas esta é uma espécie considerada sinônimo de Leucoagaricus gongylophorus. No Brasil, existe apenas um registro de fungo encontrado em ninho ativo de formiga na natureza: Leucoagaricus gongylophorus (Spielmann \& Putzke 1998). Algumas espécies de Squamanita Imbach são micoparasitas sobre Galerina, Cystoderma Fayod e Phaeolepiota Maire ex Konrad \& Maublanc (Redhead et al. 1994).

Ao contrário do que considerou Pereira \& Putzke (1989), há referência de algumas espécies de Agaricaceae como formadoras de micorrizas (Smith \& Read 2008), como Lepiota subgracilis Kühner com Salix repens (Angiospermae); e também Macrolepiota rachodes (Vittad.) Singer com Picea abies, Pinus sylvestris e P. virginiana (Gymnospermae) e $M$. procera (Scop.) Singer com Larix decidua e Pinus sylvestris (Gymnospermae), e com as Angiospermae Quercus faginea e $Q$. suber (Trappe 1962).

\section{Sistemática da família Agaricaceae Fr.}

\section{Os gêneros agaricoides}

No início da história da classificação dos fungos, Agaricaceae sensu Fries (1821, 1874) eram todos os fungos macroscópicos com o himênio lamelado. Fries (1821), Saccardo (1887) e Massee (1902) consideraram que a divisão primária dependia da cor dos esporos observados em deposição numa superfície opaca, não observados em microscópio óptico. Estes grupos eram conhecidos comumente como "seções", em número de cinco:

- Leucosporae: nesta "seção", eram colocados os fungos com "esporos" brancos ou levemente amarelos ou rosados.

- Chlorosporae: aqui, foram colocadas as espécies de "esporos" verdes claros a até verdeazulados.

- Rhodosporae: salmão e rosa eram as cores que predominavam nesta "seção".

- Ochrosporae: nesta, se reuniam todos que possuíam "esporos" ocráceos a ferrugíneos.

- Melanosporae: aqui estavam todas as espécies de "esporos" pretos, marrons enegrecidos ou pretos com tons purpúreos.

Outros micólogos do início do século XX, como Kauffman (1918), dividiam os representantes desta família friesiana de acordo com a cor da esporada, mas não nomeava estes grupos como Saccardo (1887) ou Massee (1902) o fizeram. Maire (1902), ao contrário, considerava a ordem Agaricinées com as famílias Hygrophoracées, Russulacées, Paxilacées, Boletacées e Agaricacées. Esta última, então, possuía as tribos Clitocybées, Tricholomées, Goniosporées, Amanitées, Pholiotées e Coprinés. Já para Gäumann \& Dodge (1928), Agaricales possuía as famílias Coprinaceae, Lactariaceae, Hygrophoraceae, Paxillaceae, Boletaceae e Agaricaceae, o qual esta última possuía as tribos Schizophyllae, Marasmieae, Trichoilomateae, Clitocybeae e Amaniteae. Sendo assim, a tribo Amaniteae continha, entre outros, os gêneros Lepiota (Pers.) Gray e Psalliota (Fr.) P. Kumm. (sinônimo de Agaricus).

O primeiro a nomear a ordem de acordo com o Código Internacional de Botânica foi Clements \& Shear (1932), mas considerando as "seções" apresentadas anteriormente. Já Singer (1936) tratava Agaricales em 14 famílias, entre elas Leucocoprinaceae com as tribos Leucocoprinae, contendo Leucocoprinus Pat. (= Macrolepiota) e Hiatula Fr., e Lepiotae, com os gêneros Cystoderma, Lepiota, Lepiotella (E.-J.Gilbert) Konrad e Chlorophyllum.

Mas foi com Singer (1949) que a ordem começou a ganhar a configuração atual, e as famílias foram definidas, entre elas Agaricaceae, com 15 gêneros divididos em 4 tribos: 
1. Tribo Leucocoprinae Singer: Clarkeinda Kuntze, Chlorophyllum, Macrolepiota, Leucoagaricus (Locq.) ex Singer e Leucocoprinus Pat.

2. Tribo Agariceae Pat.: Agaricus, Melanophyllum Velen. e Cystoagaricus Singer.

3. Tribo Lepiotae: Pseudobaeospora Singer e Lepiota.

4. Tribo Cystodermatae: Drosella Maire, Smithiomyces Singer, Cystoderma Fayod, Phaeolepiota e Ripartitella Singer.

As características que separavam as tribos Leucocoprinae e Agariceae continuaram as mesmas nas edições posteriores (Singer 1962, 1986), mas entre Lepiotae e Cystodermatae uma característica muito importante foi ressaltada: os basidiosporos da primeira eram essencialmente dextrinóides e da segunda inamilóides ou amilóides.

Para Smith (1973), os gêneros de Agaricaceae estavam divididos em outras duas famílias:

- Lepiotaceae Roze: Clarkeinda, Chlorophyllum, Volvolepiota Singer, Lepiota e Leucocoprinus.

- Agaricaceae Fr.: Agaricus, Melanophyllum e Cystoagaricus.

Este autor tratava Macrolepiota e Leucoagaricus sinônimos de Leucocoprinus, não considerando o fato da margem plicada-sulcada como caráter taxonômico para a distinção dos gêneros. A divisão entre Agaricaceae e Lepiotaceae continuou sendo aceita por Redhead (1987), entretanto, Leucocoprinus, Macrolepiota e Leucoagaricus reaparecem como gêneros distintos, juntamente com Cystolepiota Singer, Lepiota, Chlorophyllum e Pseudobaeospora na família Lepiotaceae.

A composição desta família não mudou muito desde Singer (1949); apenas na sinonimização de Drosella com Chamaemyces Battarra ex Earle e a inclusão de alguns novos gêneros. Deste modo, Singer (1986) considera Agaricaceae Fr. com 25 gêneros distribuídos em 4 tribos:

- Tribo Leucocoprinae Singer: Clarkeinda, Clorophyllum, Volvolepiota, Macrolepiota, Leucoagaricus, Leucocoprinus e Sericeomyces Heinem.

- Tribo Agariceae Pat.: Agaricus, Cystoagaricus, Crucispora E. Horak, Melanophyllum e Micropsalliota Höhn.

- Tribo Lepiotae Fayod: Smithiomyces Singer, Hiatulopsis Singer \& Grinling, Janauaria Singer, Cystolepiota, Lepiota (Pers.) Gray e Chamaemyces.

- Tribo Cystodermatae: Cystoderma, Phaeolepiota Maire ex Konrad \& Maublanc, Dissoderma (A.H. Sm. \& Singer) Singer, Squamanita Imbach, Pseudobaespora Singer, Ripartitella Singer e Horakia Oberw.

O dicionário micológico de Hawksworth et al. (1995) tratava a família com 42 gêneros: Agaricus, Arenicola Velen., Chamaemyces, Chlorolepiota Sathe \& S.D. Deshp., Chlorophyllum, Clarkeinda, Constricta R. Heim \& Mel.-Howell, Crucispora, Cystoagaricus, Cystoderma, Cystolepiota, Dissoderma (A.H. Sm. \& Singer) Singer, Graminicola Velen., Hiatulopsis Singer \& Grinling, Hymenagaricus Heinem., Janauaria Singer, Lepiota, Leucoagaricus, Leucocoprinus, Macrolepiota, Melanophyllum, Metraria Cooke \& Massee, Metrodia Raithelh., Micropsalliota, Morobia E. Horak, Mycenopsis Velen., Phaeopholiota Locq. \& Sarwal, Phlebomema R. Heim., Pseudoauricularia Kobayasi, Pseudobaeospora, Pulverolepiota Bon, Rugosospora Heinem, Schinzinia Fayod, Sericeomyces, Singerina Sathe \& S.D. Deshp., Smithiomyces, Squamanita, Stylobates Fr., Termiticola E. Horak, Valentinia Velen., Verrucospora E. Horak (=Horakia) e Volvolepiota.

Com o avanço da sistemática filogenética, aparecem propostas de reorganizar a família Agaricaceae. Os primeiros foram Johnson \& Vilgalys (1998), que propuseram a exclusão da tribo Cystodermatae e a retenção das demais. Outras considerações destes autores foram a 
transferência de Coprinus seção Comati e os gêneros secotióides Montagnea Fr. e Podaxis Desv. para a família em questão (Redhead et al. 2001).

O dicionário micológico elaborado por Kirk et al. (2001), considerava, em parte, o trabalho de Johnson \& Vilgalys (1998) e lista 51 gêneros e 918 espécies de Agaricaceae, incluindo vários gêneros de basidioma secotióide e gasteróides, como podemos observar abaixo:

1. Agaricus: 200 espécies.

2. Allopsalliota Nauta \& Bas: 1 espécie, Holanda.

3. Araneosa Long: 1 espécie, EUA.

4. Chamaemyces: 1 espécie

5. Clarkeinda: 5 espécies.

6. Clavogaster Henn.: 1 espécie, Nova Zelândia.

7. Chlorolepiota: 1 espécie, Índia.

8. Chlorophyllum: 6 espécies.

9. Constricta: 1 espécie, Costa do Marfim.

10. Crucispora: 2 espécies, Nova Zelândia e Ásia.

11. Cystoagaricus: 4 espécies.

12. Cystolepiota: 10 espécies.

13. Endolepiota Singer: 1 espécie, Argentina. Basidioma gasteroide.

14. Endoptychum Czern.: 7 espécies. Basidioma gasteroide.

15. Gasterellopsis Routien: 1 espécie, EUA.

16. Gymnogaster J.W. Cribb: 1 espécie, Austrália.

17. Gyrophragmium Mont.: 5 espécies.

18. Heinemannomyces Watling: 1 espécie, Malásia.

19. Hiatulopsis: 2 espécies, Congo e Brasil.

20. Holocotylon Lloyd: 3 espécies.

21. Hymenagaricus: 10 espécies.

22. Hypogaea E. Horak: 1 espécie, Argentina. Basidioma gasteroide.

23. Janauaria: 1 espécie, Brasil.

24. Lepiota: 400 espécies.

25. Leucoagaricus: 75 espécies.

26. Leucocoprinus: 40 espécies.

27. Longula Zeller: 1 espécie, América do Norte. Basidioma gasteroide.

28. Macrolepiota: 30 espécies.

29. Melanophyllum: 3 espécies.

30. Metraria: 2 espécies.

31. Metrodia: 2 espécies. Argentina

32. Micropsalliota: 40 espécies.

33. Montagnea Fr.: 5 espécies. Áreas secas subtropicais.

34. Neosecotium Singer \& A.H. Sm.: 2 espécies. Basidioma gasteroide.

35. Notholepiota: 1 espécie, Nova Zelândia. Basidioma gasteroide.

36. Panaeolopsis Singer: 4 espécies. Basidioma gasteroide.

37. Phaeopholiota: 1 espécie.

38. Phlebomema: 1 espécie, Madagascar.

39. Phyllogaster Pegler: 1 espécie, Gana. Basidioma gasteroide.

40. Podaxis Desv.: 10 espécies.

41. Pseudoauricularia: 1 espécie, Papua Nova Guiné.

42. Schinzinia: 1 espécie, Oeste da África.

43. Secotium Kunze: 10 espécies.

44. Singerina: 1 espécie, Índia.

45. Smithiogaster J.E. Wright: 1 espécie, Argentina. Basidioma gasteroide.

46. Smithiomyces: 2 espécies.

47. Termiticola: 1 espécie, Papua Nova Guiné. 
48. Verrucospora: 1 espécie, África tropical.

49. Volvigerum (E. Horak \& M.M. Moser) R. Heim: 1 espécie, Nova Zelândia.

50. Volvolepiota: 2 espécies, Brasil e Argentina.

51. Xanthagaricus (Heinem.) Little Flower, Hosag \& T.K. Abraham: 12 espécies.

Dos representantes da tribo Cystodermatae de Singer (1986), Cystoderma, Pseudobaespora, Ripartitella e Squamanita e Phaelepiota foram excluídas de Agaricaceae e introduzidas em Tricholomataceae, e Dissoderma foi sinonimizado com Squamanita (Kirk et al. 2001). Apenas Horakia (como Verrucospora) continuava na família (Kirk et al. 2001).

Os gêneros verdadeiramente agaricoides considerados por Kirk et al. (2001), como Termiticola e Verrucospora, também possuem posição taxonômica discutível. O primeiro foi descrito originalmente para Papua Nova Guiné (Horak 1979), e foi sinonimizado com Agaricus, seção Laeticolores Heinem. do subgênero Lanagaricus Heinem., por possuir véu universal remanescente no píleo e estipe (Singer 1986), mas para Vellinga (2004), este é um gênero realmente independente pertencendo ao clado Leucoagaricus / Leucocoprinus. O segundo, conhecido para o leste da África (Pegler 1977) foi sinonimizado com Horakia Oberw., mas considerado como Telephoraceae pela forma dos basidiosporos (Oberwinkler 1976). Segundo Singer (1986), apesar de Verrucospora de ter sido corretamente renomeado, os agaricólogos não devem considerar Horakia como representante dos Telephorineae simplesmente pela forma dos basidiosporos.

Metraria foi revisado por Singer (1955) apresentando basidiosporos punctados, muito próximo de várias espécies de Crepidotus e Porphyrellus e basidioma do tipo-Amanita. Ele conclui que Metraria pode ser um gênero independente, mas se pertencer à Amanitaceae seria diferente de Amanita e Limacella pela ornamentação dos basdiosporos.

Phlebonema é um gênero monoespecífico descrito para a África, necessitando ser observado o comportamento dos basidiosporos com os reagentes comumente usados em Agaricaceae, e o problema da posição taxonômica desse gênero pode ser resolvido com novas coletas (Singer 1986). Consntricta também foi descrito para a África e possui basidiosporos esféricos, verrugosos e amilóides, e basídios polimórficos (Meléndez-Howell 1965).

\section{Os "Gasteromycetes" da família Agaricaceae}

Os fungos de basidioma secotioide e gasteroide, por muito tempo já eram considerados próximos dos Agaricales, partir de observações no desenvolvimento, morfologia e microscopia (Conrad 1915; Cunningham 1926; Morse 1933; Barnett 1943; Zeller 1943; Singer 1958; Singer \& Smith 1960; Saville 1968; Heim 1971; Thiers 1984; Agerer 2002). Holm (1949) e Singer (1958) afirmaram que os fungos agaricoides derivaram de um gasteromiceto ancestral, ao contrário de Heim (1971) e Thiers (1984), que afirmaram que a evolução teve o caminho oposto. Assim, Reijnders (2000) relatou que as linhas evolucionárias no passado distante continuam desconhecidas, e com os estudos modernos possivelmente se obterá uma resposta concreta. No entanto, o basidioma agaricoide é bastante antigo, sendo datado desde o Cretáceo (Hibbett et al. 1995, 1997a; Heads et al. 2017a,b).

Com o advento da filogenia molecular, a relação entre fungos agaricoides, secotioides e gasteroides começou a ser elucidada. Um dos trabalhos pioneiros foi feito por Bruns et al. (1989), relatando como o gênero Rhizopogon evoluiu a partir de um fungo suiloide ancestral. Depois deste, outros estudos com base molecular comprovam o relacionamento dos fungos de basidioma agaricoides com secotioides e gasteroides (Baura et al. 1992; Hopple \& Vilgalys 1994, 1999; Kretzer \& Bruns 1997; Hibbett et al. 1997b; Johnson \& Vilgalys 1998; Martin et al. 1999; Miller et al. 2000; Grubisha et al. 2001; Krüger et al. 2001; Peinter et al. 2001; Moncalvo et al. 2002; Vellinga 2003, 2004; Vellinga et al. 2003).

Os primeiros autores a tratarem os gêneros secotioides e gasteroides dentro da família Agaricaceae a partir de análise molecular e filogenética foram Johnson \& Vilgalys (1998), com 
Montagnea e Podaxis. Kirk et al. (2001) transferiram outros gêneros de basidiomas secotióides e gasteroides para Agaricaceae.

Alguns exemplos de fungos secotioides e gasteroides, e o grupo taxonômico para os quais foram originalmente descritos podem ser citados:

Araneosa, um gênero gasteroide típico descrito originalmente para a família Arachniaceae. Este se caracteriza pelo esporófito estipitado e pela columela, ao contrário de Arachnion Schwein., que é séssil (Long 1941). Ambos já foram classificados na família Lycoperdaceae.

Outro gênero gasteroide incluído em Agaricaceae por Kirk et al. (2001) é Gasterelopsis, e, apesar de ter hábito gasteróide, a gleba se dissolve, como o píleo de Coprinus apesar de não ser na forma líquida (Routhien 1940). Um gênero próximo a ele é Gasterella Zeller \& L.B. Walker, um fungo epígeo e muito pequeno, medindo 200-700 $\mu \mathrm{m}$ de diâmetro (Zeller \& Walker 1935), acomodada dentro de Gasterellaceae (Zeller 1948).

Em Agaricaceae também são considerados gêneros de basidioma secotioides, os quais já eram anteriormente comparados aos fungos de basidioma agaricoide por observações desenvolvimento inicial dos basidiomas (Conrad 1915; Morse 1933; Barnett 1943; Zeller 1943) ou na ornamentação dos basidiosporos (Singer \& Smith 1960).

Holocotylon, por exemplo, possui basidioma subgloboso sem uma base estéril, o perídio é facilmente destacado e o himênio é composto por uma paliçada compacta de paráfises, conidióforos e basídios; o capilício está ausente e o basidiosporo é esférico a elipsóide (Zeller 1947). Segundo este autor, Holocotylon lembra o gênero Arachnion e, relatou que a presença de conidiosporos não é comum em Gasteromycetes. Zeller (1947) também relatou que o posicionamento taxonômico deste gênero é obscuro, pois apesar de sua deiscência ser do tipoCalvatia, a manutenção da gleba na maturidade fez este autor incluir Holocotylon em Hymenogastraceae.

Outro gênero secotoide agora um Agaricaceae é Longula, considerado ser o nome correto de Longia, já que este é um nome de gênero de Uredinales (Zeller 1945). Para Zeller (1943) a distinção de Longia (=Longula) com o outro gênero secotióide desta família, Gyrophragmium é paralela da feita entre Lepiota e Amanita em relação à presença de volva, pois assim com Lepiota, Longula não apresenta volva.

Volvigerum foi descrito para a Nova Zelândia por Heim (1966) e é caracterizado por também possuir uma volva bem distinta, pelos basidiosporos verrucosos e pela natureza lameliforme de seu himênio.

Podaxis, Montagnea e Panaeolopsis são outros gêneros secotióides considerados Agaricaceae; o primeiro e o segundo são mais conhecidos como representantes de Podaxaceae (Podaxales, Gasteromycetes), como consideraram, por exemplo, McKnight (1985), Baseia \& Galvão (2002) e Baseia \& Milanez (2002); o terceiro foi descrito por Singer (1976) para a família Montagneaceae Singer, juntamente com Montagnea e Polyplocium Berk.

Para Moncalvo et al. (2002), os verdadeiros "puffballs" (Lycoperdaceae) e Tulostomataceae também pertencem à família Agaricaceae, resultado confirmado por Vellinga (2004).

\section{Agaricaceae no conceito moderno}

Depois de Singer (1986), outras mudanças ocorreram, principalmente nos fungos lepiotóides. Além da exclusão da tribo Cystodermatae (Johnson \& Vilgalis 1998), a redefinição de Rugosospora por Guzmán et al. (1989) e Franco-Molano (1995); a sinonimização de Sericeomyces com Leucoagaricus (Vellinga 2000), de Volvolepiota com Macrolepiota (Vellinga \& Yang 2003), e do grupo Macrolepiota rachodes com Chlorophyllum (Vellinga 2003; Vellinga et al. 2003) também são mudanças significativas que ocorreram após Singer (1986). Vellinga (2004) transferiu Cystoagaricus para Psathyrellaceae e exclui Leucopholiota da família. Estudos recentes de Örstadius et al. (2015) corroboraram com esta descoberta.

Deste modo, Vellinga (2004) considerou os gêneros de basidioma agaricóide, secotióide e gasteróide divididos dentro de Agaricaceae em 10 clados: clado Agaricus, com Agaricus, 
Micropsalliota e Allopsalliota; clado Chlorophyllum; clado Macrolepiota; clado Leucoagaricus /Leucocoprinus, com Sericeomyces e os fungos cultivados por formigas; clado Lepiota, com Lepiota de um lado, e uma combinação de Cystolepiota, Echinoderma, Melanophyllum e Pulverolepiota, do outro; clado Podaxis; clado Lycoperdaceae, com Bovista, Lycoperdon, Morganella e Clavatia; clado Chamaemyces; clado Tulostomataceae, com Tulostoma e Battarraea; e clado Coprinus comatus, com Coprinus e Montagnea.

Matheny et al. (2006), a partir de análise filogenética multigene, reconheceu a ordem Agaricales com seis grandes clados: 'plicaturopsidoide', 'pluteoide', 'higroforoide', 'marasmioide', 'tricholomatoide' e 'agaricoide'. Deste modo, o clado 'adagicoide' foi considerado como contendo várias famílias com representantes de esporada escura, incluindo Agaricaceae. Também observaram que o gênero Cystoderma, juntamento com o gênero gasteroide Cyathus Haller, ficou filogeneticamente de posição basal em relação aos demais gêneros desta família, e consideraram para Cystoderma a família Cystodermataceae.

Recentemente, Vellinga et al. (2011) descreveram dois gêneros novos de Agaricaceae de esporada clara, Coniolepiota Vellinga e Eriocybe Vellinga com base em dados moleculares/filogenéticos e morfológicos.

\section{Ocorrência de Agaricaceae de basidioma agaricoide no Brasil}

Os registros de Agaricaceae agaricoides mais antigos para o Brasil são encontrados nos trabalhos de Berkeley (1843, 1856a,b, 1879-1880) e Montagne (1856), onde várias espécies de Agaricus sensu lato são referidas, e Spegazzini (1889), com Pleurotus brasiliensis Speg., considerado basinômio de Ripartitella brasiliensis (Speg.) Singer (Singer 1950).

No início do século XX surgiram os trabalhos de Rick (1905, 1906, 1907, 1908, 1920, 1926, 1930, 1937, 1938, 1939) onde muitas espécies de Agaricaceae são citadas para o Rio Grande do Sul, reunidas em seu trabalho póstumo, publicado pelo Padre Balduíno Rambo (Rick 1961), que citou os gêneros Lepiota (Pers.) Gray, Lepiotella (=Volvolepiota), Schulzeria Bres. \& Schulzer (=Lepiota sem anel) e Psalliota (=Agaricus L. ex Fr.). Lepiota sensu lato foi o gênero mais representativo, com 93 espécies citadas, divididas em quatro seções.

Na Seção Procerae, as quais estão reunidas as espécies com pileo maiores que $10 \mathrm{~cm}$, temos como representantes $L$. aureoconspersa Rick, L. badhamii (Berk. \& Bromme) Quél., $L$. bonaerensis Speg., $L$. brinkmanii Rick, $L$. coriacea Rick, L. excoriata (Schaeff.) P. Kumm., $L$. molybdites (G. Mey.) Sacc., L. morgani (Peck) Sacc., L. permita Barla, Lepiota procera (Scop.) Gray, L. rhacodes (Vittad.) Quél., L. stercoraria Rick e L. zeyheri Berk. Contudo, vários destes nomes estão considerados em Chlorophyllum ou Macrolepiota (Vellinga et al. 2003a,b).

Na Seção Mediae, com pileo 5-10 cm e basidiosporos 5-10 $\mu \mathrm{m}$, encontra-se L. clypeolaria (Bull.) P. Kumm., L. cyanea Rick, L. denticulata Speg., L. erythrella Speg., L. forquignonii, $L$. friesii (Lasch) Quél., L. fuscoquamea Peck, $L$. hispida Gillet., $L$. ingrata, L. leviceps Speg., $L$. medularis Rick [= Chamaemyces medularis (Rick) Raithelh], L. meleagris (Sowerby) Quél., $L$. pteropoda Kalchbr. \& MacOwan, L. rickiana Speg. e L. steinhausii (Penz.) Sacc.

A Seção Minores, contém representantes com o pileo variando entre $3-5 \mathrm{~cm}$, com as seguintes espécies: L. cepaestipes (Sowerby ex Fr.) Quél. (mais 8 variedades), L. metulispora (Berk. \& Broome) Sacc., L. cristata (Bolton) P. Kumm., L. licmophora (Berk. \& Broome) Sacc., $L$. sulphurina Clem., L. longistriata Peck, $L$. felinoides Peck, L. olivaceommamosa Rick, $L$. rubrosquamosa Rick, L. holosericea (Fr.) Gillet, L. atrocoerula Rick, L. bulbipes (Mont.) Sacc., $L$. flavosericea Rick, $L$. serenula P. Karst., L. felina (Pers.) P. Karst. e $L$. sordida Rick.

A Secão mais numerosa é a Minimae, composta por 51 espécies, a saber: L. albosquamosa Rick, L. anceps Rick, L. anthomyces Berk. \& Broome, L. apalochroa (Berk. \& Broome) Sacc., $L$. atrorupta Rick, L. aurantiaca Henn., L. brunnescens Peck, L. brunneoannulata Rick, $L$. brunneopurpurea Rick, L. brunneosquarrosa Rick, L. citrinella Speg., L. confusa Rick, L. cristatula Rick, $L$. delicata (Fr.) P. Kumm., L. dubia Rick, $L$. erminea (Fr.) P. Kumm., L. flavipes Rick, L. fulvastra Berk. \& M.A. Curtis, $L$. fulvolutea Rick, $L$. gracilis Peck, L. grisea Rick, $L$. hypholoma Rick, $L$. inclinata Rick, L. lanosofarinosa Rick, L. lugens Rick, L. micropholis (Berk. \& 
Broome) Sacc., L. noctiphila (Ellis) Sacc., L. parvannulata (Lasch) Gillet, L. phaeopus Rick, L. plumbicolor Berk. \& Broome, L. pratensis Speg., L. proletaria Rick, L. pusilla Speg., L. pyrrhaes Berk. \& Broome, L. radicata Rick, L. revoluta Rick, L. rosella Rick, L. rubella Bres., L. rubrostraminea Rick, rufogranulata Henn., L. rupta Rick, L. russoceps Berk. \& Broome, L. seminuda (Lasch) Gillet, L. serrulata Rick, L. sulphureosquamosa Rick, L. straminea Rick, L. tortipes Rick, .L. trombophora Brek. \& Broome e L. unicolor Rick.

O gênero Agaricus também é bem representado nos trabalhos de Rick (1939, 1961), sob o nome de seu sinônimo Psalliota com as espécies $P$. abruptibulba (Peck) Rick, P. albovelutina Rick, $P$. argentina (Speg.) Herter, $P$. californica (Peck) Rick, $P$. campestris (L.) Gillet, $P$. comtula (Fr.) Quél., P. cretacea (Fr.) Gillet., P. cretacella (G.F. Atk.) Rick, $P$. echinata (Roth) P. Kumm., $P$. elvensis Berk. \& Broome, P. marcelina Rick, P. perrara (Schulzer) Rick, P. platomyces (=placomyces) (Peck) Henn., P. pratensis (Schaeff.) Gillet, P. rhodochroa (Berk. \& Broome) Rick, P. silvatica (Schaeff.) P. Kumm., P. straminea Rick, P. subrufescens (Peck) Kauffman, $P$. trissulphurata (Berk.) Rick e P. villatica (Brond.) Bres.

Theissen (1912) também citou Agaricaceae para o Rio Grande do Sul, com Lepiota cepaestipes, L. licmophora (Berk. \& Broome) Sacc., L. gracilis Peck e L. fuscoroseola Speg. para este estado.

O primeiro a revisar as espécies de Agaricaceae no Rio Grande do Sul publicadas por Rick foi Singer (1953), confirmando Agaricus campestris ou pampeanus (Speg.) Singer, Chlorophyllum molybdites, Lepiota crassior Singer, L. flavidula (Rick) Singer, Lepiotella brunnea Rick, Leucoagaricus confusus (Rick) Singer, L. rubrosquamosus (Rick) Singer, L. olivaceomamillatus (Rick) Singer, Leucocoprinus cepaestipes (Sowerby) Pat., Macrolepiota bonaerensis (Speg.) Singer, Melanophyllum echinatum (Roth ex Fr.) Singer, Ripartitella brasiliensis (Speg.) Singer. e Smithiomyces mexicanus (Murrill) Singer.

Posteriormente, Heim (1957) descreveu o experimento de Alfred Møller, onde cita para Blumenau, Santa Catarina Rozites gongylophora A. Møller em ninho de formiga, sinonimizado então para Leucocoprinus gongylophorus. Jezek (1973) citou Agaricus xanthodermus Genev. Macrolepiota mastidea e descreveu Leucocoprinus nigricans Jezek para o estado do Rio de Janeiro.

Singer (1973) descreveu Lepiota xanthophylla Singer non. P.D. Orton para o estado do Pará e Singer (1986, 1989) Cystolepiota albogiva Singer, C. amazonica Singer, C. potassiovirens Singer, Chamaemyces paraensis Singer, Hiatulopsis aureoflava Singer, Janauaria amazônica Singer, Lepiota izonetae Singer, L. xanthophylloides Singer (nome novo para ' $L$. xanthophylla'descrito em Singer 1973) e Leucoagaricus tricolor Singer para Amazonas e Pará.

Para o estado de São Paulo há vários registros de Agaricaceae; Bononi et al. (1981a) citaram Lepiota aurea Pers., L. clypeolaria, L. morgani (= Chlorophyllum molybdites), Leucocoprinus cepaestipes, L. fragilissimus (Ravenel) Pat., Phaeolepiota aurea (Matt.) Maire e Volvolepiota brunnea (Rick) Singer; Bononi et al. (1981b) referiram a Leucocoprinus gongylophorus; Grandi et al. (1984) cita Agaricus campestris, A. porosporus Heinem., A. singeri Heinem., Chlorophyllum molybdites, Lepiota lentiginosa Pegler, L. epicharis var. occidentalis Dennis, Leucoagaricus naucinus, Leucocoprinus birmbaumii (Corda) Singer, Macrolepiota dolichaula (Berk. \& Broome) Pegler, M. mastoidea (Fr.) Singer e Ripartitella brasiliensis. No mesmo ano, Bononi et al. (1984) registraram Agaricus silvaticus Schaeff., Leucocoprinus cepaestipes (Sowerby) Pat. e Macrolepiota procera (Scop.) Singer.

No Rio Grande do Sul, Raithelhuber (1987a,b) citou Leucocoprinus bulbipes (Mont.) Raithelh., L. fulvaster (Berk. \& M.A. Curtis) Raithelh., L. inflatus Raithelh., L. noctiphilus sensu Rick, L. phaeopus (Rick) Raithelh., L. proletarius (Rick) Raithelh., L. revolutus (Rick) Raithelh., L. rivulosus Raithelh., L. russoceps (Berk. \& Broome) Raithelh., Macrolepiota bonaerensis, M. brasiliensis (Rick) Raithelh., $M$. excoriata (Schaeff.) M.M. Moser, M. gracilenta var. acuteoumbonata Raithelh., M. rhacodes (Vittad.) Singer, M. stercoraria (Rick) Raithelh. e $M$. zeyheri (Fr.) Singer.

Em seu trabalho de revisão de exsicatas depositadas nos Herbários BAFG (Buenos Aires, Argentina) e PACA (São Leopoldo, Rio Grande do Sul), Raithelhuber (1988) referiu 
Chamaemyces medularis, Lepiota olivaceomammosa var. irritans Raithelh., Leucoagaricus erythrellus (Speg.) Singer, Leucocoprinus fibrilosus Raithel., L. meleagris (Sowerby ss. Rick) Raithelh., e Smithiomyces lanosofarinosa (Rick) Raithelh. para o Rio Grande do Sul.

Para Rondônia, Capelari \& Maziero (1988) referiram Agaricus cf. silvaticus, Lepiota citriodora Dennis, L. guatopoensis Dennis, L. ochraceoaurantiaca Dennis, L. cf. phaeosticta Morgan, L. rubrotincta Peck, Leucocoprinus brebissonii (Godey) Locq., L. cepaestipes e L. fragilissimus.

Heinemann (1989) citou Micropsalliota pruinosa Heinem. e M. roseovinacea para o Rio de Janeiro; Heinemann (1993) refere Agaricus agriopotamicus Speg. para o estado do Mato grosso; A. spissicaulis F.H. Møller, A. dicystis Heinem., A. cf. fuscofibrilosus (F.H. Møller) Pilát, $A$. junquitensis Heinem., A. parasilvaticus Heinem., A. silvaticus, A. cheilotulus Heinem., $A$. cf. nivensis (F.H. Møller) F.H. Møller, $A$. cf. ochroscens Heinem. \& Goos.-Font., $A$. silvicola (Vittad.) Peck, A. meijeri Heinem., A. volvatulus Heinem. \& Goos.-Font., $A$. riberaltensis Heinem., $A$. trisulphuratus Berk., A. bugandensis Pegler, A. cf. rhopalopodius Pat., A. sulcatellus Heinem., Micropsalliota arginea (Berk. \& Broome) Pegler \& Rayner, M. cf. campestroides (Heinem.) Heinem. e $M$. cephalocystis (Heinem.) Heinem. para o Paraná; A. volvatulus Heinem. \& Goos.Font. e $A$. cf. rhopalopodius Pat. para o Rio de Janeiro e A. blazei, Murrill para São Paulo.

Na revisão dos Agaricales de São Paulo, Pegler (1997) citou Chlorophyllum molybdites, Macrolepiota bonaerensis, Leucoagaricus hortensis (Murrill) Pegler, L. imperialis (Speg.) Pegler, Leucocoprinus birmbaumii, L. sulphurellus Pegler [= Leucoagaricus sulphurellus (Pegler) B.P. Akers], L. fragilissimus, L. brebissonii, L. venezuelanus Pegler, Agaricus agyropotamycus Speg., A. porosporus Heinem. A. silvaticus, A. parasilvaticus Heinem., A. fiardii Pegler, A. hornei Murrill, A. endoxantus Berk. \& Broome, A. purpurellus (F.H. Møller) F.H. Møller, A. violaceos Baker \& Dale, A. ochraceosquamulosus Heinem., A. rufoaurantiacus Heinem., A. denisii Heinem., A. puttemansii Pegler, Micropsalliota roseovinacea Pegler, Cystolepiota marthae Singer, Lepiota subflavescens Murrill, L. lilacea Bres., L. epicharis var. occidentalis, L. lineata Pegler, L. subscristata Murrill, L. lactea Murrill, L. phaeosticta Morgan, L. nigropunctata Dennis, L. rimosa Murrill, L. murinocapitata Dennis, L. guatopoensis Dennis, L. ochraceoaurantiaca Dennis, L. quinamana Dennis, L. abruptibulba Murrill, L. citriodora Dennis, L. griseorubescens Dennis, L. serena (Fr.) Sacc. (=Sericeomyces), Cystoderma amianthinum (Scop.) Fayod, $C$. siparianum (Dennis) Thoen e Ripartitella brasiliensis (Speg.) Singer.

Franco-Molano (1995), estudando sobre o gênero Rugosospora citou material examinado proveniente de coleta realizada por Rick, identificando como $R$. pseudorubiginosa (Cifuentes \& Guzmán) Guzmán \& Bandala.

Pereira (1998) descreveu 10 espécies novas do gênero Lepiota para os Estados do Rio Grande do Sul e Santa Catarina, sendo elas: L. apicepigmentata A.B. Pereira, L. araucariicola A.B. Pereira, L. bifurcata A.B. Pereira, L. brunneotabacina A.B. Pereira, L. colorada A.B. Pereira, L. conglobata A.B. Pereira, L. cutiscamosa A.B. Pereira, L. ministipitata A.B. Pereira, L. santacruzensis A.B. Pereira e L. septata A.B. Pereira. Já em Pereira (2000), em sua revisão do gênero Lepiota para o Brasil, confirmou, além destas, mais 23 espécies: L. abruptibulba Murrill, L. aspera (Pers. ex Fr.) Quél., L. brunneoannulata, L. brunneocarnea, L. brunneopurpurea, L. brunneosquarrosa, L. clypeolaria, L. cristata, L. flavipes Rick, L. forquignonii Quél., $L$. hypholoma Rick, L. incilnata Rick, L. ingrata Rick, L. izonetae Singer, L. lugens Rick, L. olivaceomammosa Rick, L. parvannulata (Lash. ex Fr.) Gillet, L. phaeopus Rick, L. phaeosticta Morgan, L. pyrrhaes Berk. \& Broome, L. radicata Rick, L. rubella Bres. e L. rubrostraminea Rick. Posteriormente, Pereira (2001) discutiu 89 epípetos específicos de Lepiota do Herbário PACA, nos quais foram excluídos do gênero, considerados nomen nudum ou de ocorrência duvidosa, por terem a exsicata muito danificada.

No século XXI, os estudos taxonômicos envolvendo Fungos da família Agaricaceae contendo basidioma agaricoide se intensificaram. Capelari \& Gimenes (2004) descreveram Leucocoprinus brunneoluteus Capelari \& Gimenes e Capelari et al. (2006) reportaram Agaricus martineziensis Heinem. para São Paulo; Albuquerque et al. (2006) referiram várias espécies de Leucocoprinus anteriormente citadas por outros autores e Drecshler-Santos et al. (2007) 
referiram L. noctiphilus (Ellis) Heinem.; e de Meijer et al. (2007) relatou com detalhes envenenamento de uma fmília causada por Chlorophyllum molybdites. Ainda para o estado do Paraná, várias espécies foram citadas, incluindo algumas novas novas para a ciência, como $A$. stijvei de Meijer e Lepiota silva-araucariae de Meijer.

No Rio Grande do Sul destacam se os trabalhos de Rother \& Silveira (2008, 2009a,b), que citaram Agaricus cf. litoralis (Wakef. \& A. Pearson) Pilát, A. porporizon P.D. Orton, A. pseudoargentinus Albertó \& J.E. Wright, A. aff. silvaticus, Chlorophyllum molybdites, Lepiota guatopoensis, Leucoagaricus lilaceus Singer, L. rubrotinctus, L. serenus (Fr.) Bom \& Boiffard, Leucocoprinus birnbaumii (Corda) Singer, L. brebissonii, L. cepistipes, L. cretaceus (Bull.: Fr.) Locq. (Figura 2), L. fragilissimus e $L$. cf. medioflavus (Boud.) Bon.

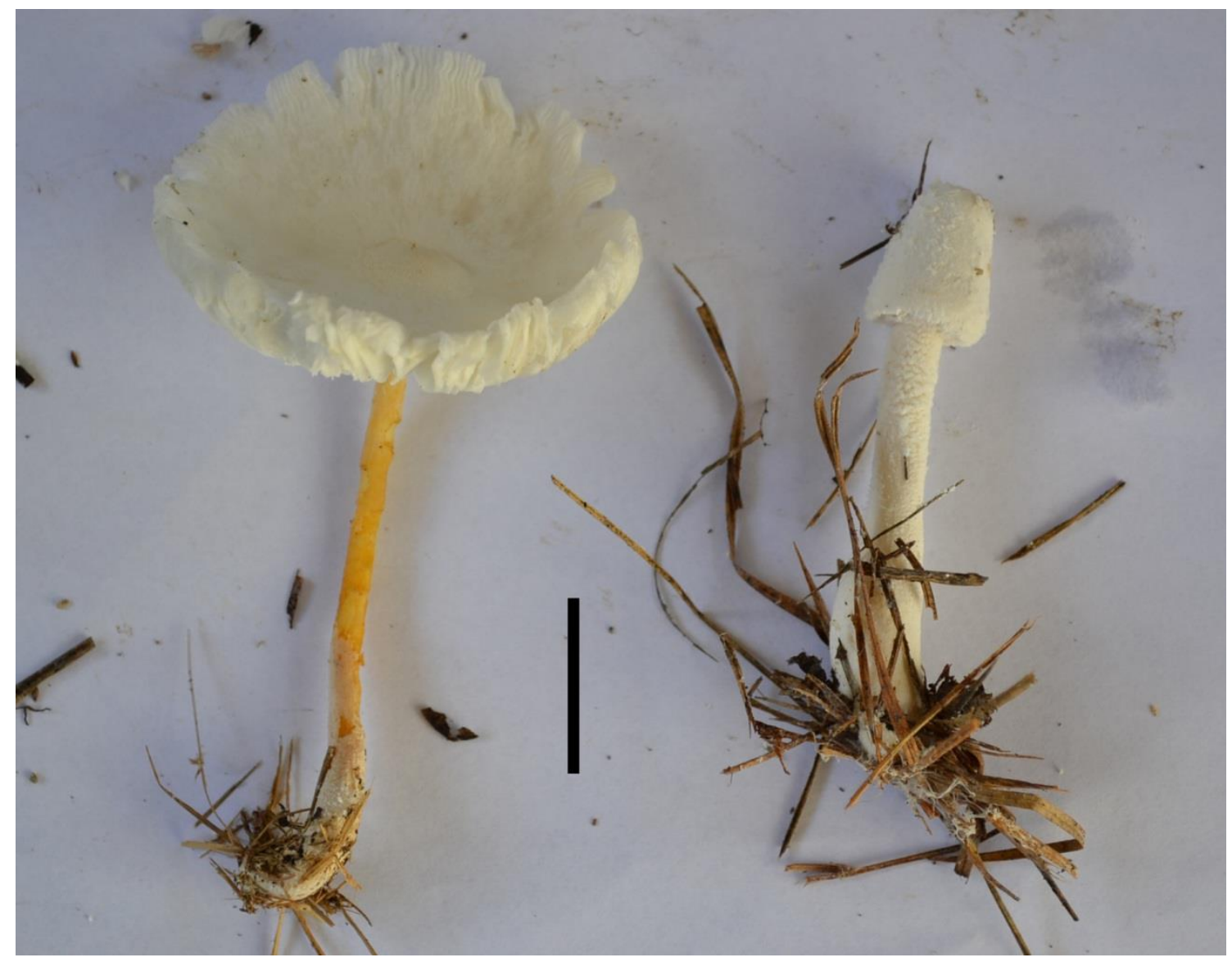

Figura 2. Leucocoprinus cretaceus. Foto: F. Wartchow.

Para o Estado do Rio de Janeiro, Albuquerque et al. (2010) citaram Agaricus dulcidulus Schulzer, A. subrufescens Peck, Cystolepiota seminuda (Lasch) Bon, Lepiota lilacea, L. echinella var. rhodorrhiza (Romang. \& Locq. ex P.D. Orton) Hardtke \& Rödel, L. forquignonii var. forquignonii Quél., L. subincarnata J.E. Lange e L. teipeitensis. Logo depois, Albuquerque \& Victória (2012) descreveram Leucocoprinus fluminensis Albuquerque \& Victoria.

Trabalhos recentes citaram mais espécies para o Brasil. Ferreira \& Cortez (2012) citaram para o estado do Paraná Lepiota elaiophylla Verbeken \& Huijser, Leucoagaricus lilaceus, $L$. rubrotinctus, Leucococprinus cretaceus, Macrolepiota colombiana Franco.-Mol. e Rugosospora pseudorubiginosa. Putzke et al. (2014) citaram 12 espécies coletadas na região central do Rio Grande do Sul, todas com potencial alimentício, a saber: Agaricus argentinus Speg., $A$. argyropotamico Speg., A. bisporus, A. campestris, A. martineziensis, A. mediofuscus (F.H. Møller) Pilát, A. subrufescens Peck, Chlorophyllum rhacodes (Vittad.) Vellinga, Macrolepiota bonaerensis, Macrolepiota colombiana, M. kerandi (Speg.) Singer e M. mastoidea. Putzke \& Putzke (2017) prepararam um checklist contendo 32 espécies de Agaricus conhecidas para o 
Brasil até esta data. Albuquerque et al. (2017) revisaram espécies de Cystolepiota, inclusive sete espécies para o Brasil: C. albogilva, C. amazonica, C. marthae, C. potassovirens, C. seminuda (Lasch) Bon e C. cistrata (Fr.) Singer ex Bon. Finalmente, Drewinski et al. descreveram $A$. globocystidiatus Drewinski \& M.A. Neves para Santa Catarina (Drewinski et al. 2017).

Existem outros trabalhos esporádicos citando Agaricaceae para alguns estados do Brasil. Muchovej et al. (1991) descreveram Leucoagaricus weberi para Minas Gerais; Heinemann \& de Meijer (1996) citaram Volvolepiota brunnea para o Paraná; Spielmann \& Putzke (1998) referem Leucoagaricus gongylophorus para o Rio Grande do Sul; e Rosa et al. (2003), citaram para Minas Gerais Agaricus cf. nigrecentulus Heinem., A. porosporus, A. cf. trinitatensis R.E.D Baker \& W.T. Dale, Leucoagaricus cf. cinereus (Quél.) Bon \& Boiffard e Leucocoprinus cf. longistriatus (Peck) H.V. Sm. \& N.S.Weber.

No nordeste brasileiro poucos trabalhos envolvendo Agaricaceae são referidos: Batista (1957), que descreveu Lepiota minuta Bat. para Pernambuco e Kimbrough et al. (1994) citaram Chlorophyllum molybdites, Lepiota erythrosticta (Berk. \& Broome) Sacc. e L. teipeitensis Murrill para o mesmo estado. Maia (1998) listou espécies de fungos encontradas na região da Mata de Dois Irmãos (Recife, Pernambuco), e citou Agaricus purpurellus, Lepiota americana Peck, Lepiota morgani e Lepiota procera Lloyd. Depois, Maia et al. (2002) citaram Agaricus brunneostictus Heinem., L. griseobrunnescens Dennis, L. holosericea (Fr.) Gillet, L. lineata Pegler, L. stuhlmannii Henn., L. americana Peck, Leucoagaricus meleagris (Sowerby) Singer, Melanophyllum sp. e Micropsalliota roseovinacea Pegler. Wartchow et al. (2008) citaram para o estado de Pernambuco Agaricus aff. parasilvaticus Heinem., A. purpurellus, A. rufoaurantiacus Heinem., Leucoagaricus sulphurellus, Leucocoprinus cretaceus, L. fragilissimus, L. elaiophylla, L. erythrosticta (Berk. \& Broome) Sacc. e Micropsalliota brunneosperma (Singer) Pegler. Finalmente, Nascimento \& Alves (2014) citaram para o semiárido do estado do Ceará Agaricus stijvei, Chlorophyllum hortense, Cystolepiota seminuda, Lepiota plumbicolor, Leucocoprinus birnmbaumii e L. fragilissimus (Berk. \& Broome) Sacc.

\section{Agradecimentos}

Aos avaliadores anônimos pela revisão crítica do manuscrito. Ao Conselho Nacional de Desenvolvimento Científico e Tecnológico (CNPq) pelas bolsas de Mestrado, Doutorado e Produtividade em Pesquisa.

\section{Referências}

Agerer R. (2002) Rhizomorph structures confirm the relationship between Lycoperdales and Agaricaceae (Hymenomycetes, Basidiomycota). Nova Hedwigia, 75: 367-385. DOI: 10.1127/0029-5035/2002/0075-0367

Akers B.P., Angels S.A. \& Kimbrough J.W. (2000) Leucoagaricus viridiflavoides, a new species from Florida, with notes on related taxa. Mycotaxon, 76: 39-50.

Albuquerque M.P. \& Victoria F.C. (2012) Leucocoprinus fluminensis (Agaricaceae, Basidiomycota), a new species from soutwest Brazilian Rain Forest. Neotropical Biology and Conservation, 7: 158-161. DOI: 10.4013/nbc.2012.73.02

Albuquerque M.P., Victoria F.C. \& Pereira A.B. (2006) Ecologia e distribuição do gênero Leucocoprinus Pat. no Rio Grande do Sul, Brasil. Acta Biologica Leopoldencia, 7: 158-161.

Albuquerque M.P., Pereira A.B. \& Carvalho-Junior A.A. (2010) A família Agaricaceae Chevall. Em trechos de Mata Atlântica da Reserva Biológica do Tinguá, Nova Iguaçu, Rio de Janeiro, Brasil: gêneros Agaricus, Cystolepiota e Lepiota. Acta Botanica Brasilica, 24: 497-509. DOI: 10.1590/S0102-33062010000200020

Albuquerque M.P., Victoria F.C., Egres C.C. \& Putzke J. (2017) Cystolepiota Singer (Agaricales) na Amazônia, Rio de Janeiro, Rio Grande do Sul (Brasil) e Tucumã (Argentina). Neotropical Biology and Conservation, 12: 48-58. DOI: 10.4013/nbc.2017.121.06

Alexopoulos C.J., Mims C.W. \& Blackwell M. (1996) Introductory mycology. $4^{\circ}$ edition. New York: John Wiley \& Sons, Inc. 869 p. 
Barnett H.L. (1943) The development and structure of Longia texensis. Mycologia, 35: 399-408.

Baseia I.G. \& Galvão T.C.O. (2002) Some interesting Gasteromycetes (Basidiomycota) in dry areas from North-eastern Brazil. Acta Botânica Brasilica, 16: 1-8. DOI: 10.1590/S010233062002000100002

Baseia I.G. \& Milanez A. (2002) Montagnea haussknechtii Rabenh. (Podaxales) a rare agaricoid fungus: first record from Brazil. Acta Botânica Brasilica, 16: 311-315. DOI: 10.1590/S010233062002000300008

Batista A.C. (1957) Alguns Agaricales saprófitos de Pernambuco. Mycopathologia et Mycologia Applicata, 8: 127-134.

Baura G., Szaro T.M. \& Bruns T.D. (1992) Gastrosuillus laricinus is a recent derivative of Suillus grevilei: molecular evidence. Mycologia, 84: 592-597.

Berkeley M.A. (1843) Notices of some Brazilian Fungi. The London Journal of Botany, 2: 629643.

Berkeley M.A. (1956a) Decades of Fungi. Decades LI-LIV. Rio Negro Fungi. Hooker's Journal of Botany and Kew Garden miscellany, 8: 129-144.

Berkeley M.A. (1956b) Decades of Fungi. Decades LI-LIV. Rio Negro Fungi (cont.). Hooker's Journal of Botany and Kew Garden Miscellany, 8: 272-280.

Berkeley M.A. (1879-1880) Fungi Brasiliensis provincia Rio de Janeiro a clar Dr. Glaziou lecti (p. 31-34). In: Warming E. (Ed.) Symbolae and floram brasiliae centralis cognocedam. Videnskabelige meddelelser fra den Naturhistoriske forening i Kjöbenhavn, 31/32.

Bononi V.L.R., Trufem S.F.B. \& Grandi R.A.P. (1981a) Fungos macroscópicos do Parque Estadual das Fontes do Ipiranga, São Paulo, Brasil, depositados no Herbário do Instituto de Botânica de São Paulo. Rickia, 9: 37-53.

Bononi V.L.R., Autuori M. \& Rocha M.B. (1981b) Leucocoprinus gongylophorus (Møller) Heim, o fungo do formigueiro de Atta sexedens rubropilosa. Rickia, 9: 93-97.

Bononi V.L.R., Mucci E.S.F., Yokomizu N.K.S. \& Guzmán G. (1984) Agaricales (Basidiomycetes) do Parque Estadual de Campos do Jordão, SP, Brasil. Rickia, 11: 85-89.

Bruns T.D., Fogel R., White T.J. \& Palmer J.D. (1989) Accelerated evolution of a false-truffle from a mushroom ancestor. Nature, 339: 140-142.

Capelari M. \& Gimenes L.J. (2004) Leucocoprinus brunneoluteus, uma nova espécie de Agaricaceae. Hoehnea, 31: 331-335.

Capelari M. \& Maziero R. (1988) Fungos macroscópicos do estado de Rondônia região dos Rios Jaru e Ji-Paraná. Hoehnea, 15: 28-36.

Capelari M., Rosa L.H. \& Lachance M.-A. (2006) Description and affinities of Agaricus martineziensis, a rare species. Fungal Diversity, 21: 11-18.

Clements F.E. \& Shear C.L. (1932) The Genera of Fungi. H.W. Wilson Co., New York.

Conrad H.S. (1915) The structure and development of Secotium agaricoides. Mycologia, 7: 94104.

Cunningham G.H. (1926) The structure and development of two New Zealand species of Secotium. Transactions of the British Mycological Society, 10: 216-224.

Dennis R.W.G. (1952) Lepiota and allied genera in Trinidad, British West Indies. Kew Bulletin, 7: 459-499.

Drechsler-Santos E.R., Pastorini L.H. \& Putzke J. (2007) Primeiro relato de fungos Agaricales em fragmento de mata nativa em Frederico Westphalen - RS. Revista Brasileira de Biociências (Suplemento 2), 5: 471-473.

Drewinski M.P., Menolli Jr.N. \& Neves M.A. (2017) Agaricus globocystidiatus: a new neotropical species with pleurocystidia in Agaricus subgen. Minoriopsis. Phytotaxa, 314:64-72.

Ferreira A.J. \& Cortez V.G. (2012) Lepiotoid Agaricaceae (Basidiomycota) from São Camiolo State Park, Paraná State, Brazil. Mycosphere, 3: 962-976. DOI: 10.5943/mycosphere/3/6/11

Fisher P.J., Stradling D.J. \& Pegler D.N. (1994) Leucoagaricus basidiomata from a live nest of the leaf-cutting ant Atta cephalotes. Mycological Research, 98: 884-888. DOI: 10.1016/S09537562(09)80259-1 
Franco-Molano A.E. (1995) Observations on Rugosospora in the Neotropics. Mycologia, 87: 574578.

Fries E.M. (1821) Systema Mycologicum 1: i-lvii. Lundae: Ex Officina Berlingiana. 520 p.

Fries E.M. (1874) Hymenomycetes Europei sive Epicisis Systematis Mycologici. Uppsala. 755 p.

Gäumann E.A. \& Dodge C.W. (1928) Comparative Morphology of Fungi. New York: McGrawHill Book Company Inc. 701 p.

Grandi R.A.P., Guzmán G. \& Bononi V.L.R. (1984) Adições às Agaricales (Basidiomycetes) do Parque Estadual das Fontes do Ipiranga, São Paulo, Brasil. Rickia, 11: 27-33.

Grubisha L.C., Trappe J.M., Molina R. \& Stapafora J.W. (2001) Biology of the ectomycorrhizal genus Rhizopogon. Phylogenetic relationships in the Boletales inferred from LSU and rDNA sequences. Mycologia, 93: 82-89. DOI: 10.2307/3761607

Guzmán G., Bandala V.M., Montoya L. \& Saldarriaga Y. (1989) Nuevas evidências sobre las relaciones micoflorísticas entre África y el neotrópico. El género Rugosospora Heinem. (Fungi, Agaricales). Brenesia, 32: 107-112.

Hawksworth D.L., Kirk D.M., Sutton B.C. \& Pegler D.N. (1995) Ainsworth \& Bisby's dictionary of the fungi. $8^{\circ}$ edition. Cambridge: CAB International. $650 \mathrm{p}$.

Heads S.W., Miler A.N., Crane J.L., Thomas M.J., Ruffatto D.M., Methven A.S., Raudabaugh D.B. \& Wang Y. (2017a) The oldest fossil mushroom. PLOS One, 12: e0178327. DOI: 10.1371/journal.pone.0178327

Heads S.W., Miller A.N. \& Crane J.L. (2017b) On the name of the oldest fossil mushroom. Mycological Progress, 16: 1071-1072. DOI: 10.1007/s11557-017-1355-4

Heim R. (1957) A propôs du Rozites gongylophora A. Møller. Revue de Mycologie, 22: 293-299.

Heim R. (1966) Breves diagnoses latinae novitatum genericarum specificarumque nuper descriptarum. Deuxiéme série. Revue de Mycologie, 31: 150-159.

Heim R. (1971) The relationships between the Agaricales and Gasteromycetes. (p. 505-534). In: Petersen R.H. (Ed.). Evolution in the Higher Basidiomycetes. Knoxville: University of Tenessee Press. $562 \mathrm{p}$.

Heinemann P. (1989) Le genre Micropsalliota en Amérique tropicale et subtropicale. Bulletin du Jardin Botanique National de Belgique, 59: 459-466.

Heinemann P. (1993) Agarici Austroamericani VIII. Agariceae des régions intertropicales d'Amérique du Sud. Bulletin du Jardin Botanique National de Belgique, 62: 355-384. Doi: $10.2307 / 3668282$

Heinemann P. \& de Meijer A.A.R. (1996) The status of Volvolepiota Singer. Bulletin du Jardin Botanique National de Belgique, 65: 405-412.

Hibbett D.S., Grimaldi D. \& Donoghue M.J. (1995) Cretaceous mushrooms in amber. Nature, 377: 487.

Hibbett D.S., Grimaldi D. \& Donoghue M.J. (1997a) Fossil mushrooms from the Miocene and Cretaceous amber and the evolition of the Homobasidiomycetes. American Journal of Botany, 84: 981-991.

Hibbett D.S., Pine E.M., Langer E., Langer G. \& Donoghue M.J. (1997b) Evolution of gilled mushrooms and puffballs inferred from ribosomal DNA sequences. Proceeding of the National Academy of Science, 94: 12002-12006.

Holm L. (1949) Some aspects on the oringin of the Gastromycetes. Svenks Botanisk Tidshrift, 43: 65-71.

Hopple Jr. J.S. \& Vilgalis R. (1994) Phylogenetic relationships among coprinoid taxa and allies based on data from restriction site mapping of nuclear rDNA. Mycologia, 86: 96-107.

Hopple Jr. J.S. \& Vilgalis R. (1999) Phylogenetic relationships in the mushroom genus Coprinus and dark-spored allies based on sequence data from the nuclear gene coding for the large ribossomal subunit RNA: divergent domains, outgroups and monophyly. Molecular Phylogenetic and Evololution, 13: 1-19.

Horak E. (1979) Three new genera of Agaricales from Papua New Guinea. Beiheft zur Sydowia, 8: 202-208.

Jezek B. (1973) Něøkolik ukàzek brasilských hub. C.C.H., 50: 75-79. 
Johnson J. \& Vilgalys R. (1998) Phylogenetic systematics of Lepiota sensu lato based on nuclear large subunit rDNA evidence. Mycologia, 90: 971-979.

Kauffman C.H. (1918) The Agaricaceae of Michigan. Michigan: Michigan Geological and Biological Survey. $924 \mathrm{p}$.

Kimbrough J.W., Alves M.H. \& Maia L.C. (1994/1995) Basidiomycetes saprófitos em troncos vivos e em folhedos de "sombreiro" (Clitoria fairchildianai [Benth.] Howard). Biológica Brasílica, 6: 51-56.

Kirk P.M., Cannon P.F., David J.C. \& Stalpers J.A. (2001) Ainsworth \& Bisby's Dictionary of Fungi. $9^{\circ}$ edition. Cambridge: CAB International. $655 \mathrm{p}$.

Kirk P.M., Cannon P.F., Minter D.W. \& Stalpers J.A. (2008) Ainsworth \& Bisby's Dictionary of Fungi. $10^{\circ}$ edition. Cambridge: CAB International. $771 \mathrm{p}$.

Kretzer A. \& Bruns T.D. (1997) Molecular revisitation of the genus Gastrosuillus. Mycologia, 89(4): 586-589.

Krüger D., Binder M., Fischer M. \& Kreiser H. (2001) The Lycoperdales. A molecular approach to the systematic of some gasteroid mushrooms. Mycologia, 93: 947-957. DOI: $10.2307 / 3761759$

Long W.H. (1941) Studies in the Gasteromycetes. III. The family Arachniaceae. Mycologia, 33: 350-355.

Maia L.C. (1998) Diversidade de fungos e liquens e sucessão fúngica na Reserva Ecológica Dois Irmãos (p. 85-113). In: Machado I.C., Lopes A.V. \& Pôrto K.C. (Eds.). Reserva Ecológica de Dois Irmãos: Estudos em um Remanescente de Mata Atlântica em Área Urbana (Recife Pernambuco - Brasil). Recife: Editora Universitária da UFPE. 326 p.

Maia L.C., Yano-Melo A.M. \& Cavalcanti M.A.Q. (2002) Diversidade de Fungos no Estado de Pernambuco (p. 15-50). In: Tabarelli M. \& Silva J.M.C. (Eds). Diagnóstico da Biodiversidade de Pernambuco. Recife: Ed. Massangana. 721 p.

Maire R. (1902) Recherches cytologique et taxonomiques sur les Basidiomycètes. These de la Faculté des Sciences de Paris. Bulletin de la Société Mycologique de France, 18: 12-209.

Martin M.P., Hogberg N. \& Llistosella J. (1999) Macowanites messapicoides, a hypogeous relative of Russula messapica. Mycological Research, 103: 203-208.

Massee G. (1902) European Fungus Flora. Agaricaceae. London: Duckworth \& Co. 274 p.

Matheny P.B., Curtis J.M., Hofstetter V., Aime M.C., Moncalvo J.-M., Ge Z.-W., Yang Z.-L., Slot J.G., Ammirati J.F., Baroni T.J., Bougher N.L., Hughes K.W., Lodge D.J., Kerrigan R.W., Seidl M.T., AAnen D.K., DeNitis M., Daniele G.M., Desjardin D.E., Kropp B.R., Norvell L.L., Parker A., Vellinga E.CC., Vilgalys R. \& Hibbett D.S. (2006) Major clades of Agaricales: a multilocus phylogenetic overview. Mycologia, 98: 982-995.

McKnight K.H. (1985) The small-spored species of Podaxis. Mycologia, 77: 24-35.

Meijer A.A.R., Amazonas M.A.L.A., Rubio G.B.G \& Curial R.M. (2007) Incidences of poisonings due Chlorophyllum molybdites in the state of Paraná, Brazil. Brazilian Archives of Biology and Technology, 50: 479-488. DOI: 10.1590/S1516-89132007000300014

Meléndez-Howell L.M. (1965) Un noveu cas de bourgeonement et de polymorphisme sporaux chez les Agarics. Revue de Mycologie, 29: 315-325.

Miller S.L., McClean T.M., Walker J.F. \& Buyek B. (2000) A molecular phylogeny of Russulaceae inclunding agaricoid, gastroid and pleurotoid taxa. Mycologia, 93: 344-354.

Moncalvo J.M., Vilgalys R., Redhead S.A., Johnson J.E., James T.Y., Aime M.C., Hofstetter V., Verduin S.J.W., Larsson E., Baroni T.J., Thorn R.G., Jacobsson S., Clémençon H. \& Miller Jr. O.K. (2002) One hundred and seventeen clades of euagarics. Molecular Phylogenetic and Evolution, 23: 357-400. DOI: 10.1016/S1055-7903(02)00027-1

Montagne J.P.F.C. (1856) Septième centurie de plantes cellulaires nouvelles, tant indigènes qu' exotiques. Annales des Sciences Naturélles, Botanique Série, 4: 333-374.

Morse E.E. (1933) A study of the genus Podaxis. Mycologia, 25: 1-33.

Muchovej J.J., Della Lucia I.M. \& Muchovej R.M.C. (1991) Leucoagaricus weberi sp. nov. from a live nest of leaf-cutting ants. Mycological Research, 95: 1308-1311. 
Nascimento C.C. \& Alves M.H. (2014) New records of Agaricaceae (Basidiomycota, Agaricales) from Araripe Nacional Forest, Ceará State, Brazil. Mycosphere, 5: 319-332.

Oberwinkler F. 1975 (1976). Eine agaricoide Gattung der Thelephorales. Sydowia, 28: 359-361.

Örstadius L., Rygberg M. \& Larsson E. (2015) Molecular phylogenetics and taxonomy in Psathyrellaceae (Agaricales) with focus on psathyrelloid species: introduction of the three new genera and 18 new species. Mycological Progress, 14: 25. DOI: 10.1007/s11557-015-1047-x

Pegler D.N. (1977) A preliminary Agaric flora of East Africa. Kew Bulletin Additional Series, 6: $1-615$.

Pegler D.N. (1997) The Agarics of São Paulo, Brazil. Kew: Royal Botanical Gardens. 68 p.

Pegle D.N. \& Rayner R.W. (1969) A contribution of the agaric flora of Kenya. Kew Bulletin, 23: 347-412.

Peinter U., Bougher N., Castellano M.A., Moncalvo J.-M., Moser M., Trappe J.M. \& Vilgalys R. (2001) Multiple origins of sequestrate fungi related to Cortinarius (Cortinariaceae). American Journal of Botany, 88: 2168-2179. DOI: 10.2307/3558378

Pereira A.B. (1998) Espécies novas do gênero Lepiota (Agaricaceae) do sul do Brasil. Iheringia, 51: $227-247$.

Pereira A.B. (2000) Contribuição ao conhecimento do gênero Lepiota no Brasil. I. Pesquisas Botânica, 50: 27-77.

Pereira A.B. (2001) Contribuição ao conhecimento do gênero Lepiota no Brasil. II. Pesquisas Botânica, 51: 7-30.

Pereira A.B. \& Putzke J. (1989) Famílias e gêneros de fungos Agaricales (cogumelos) no Rio Grande do Sul. Santa Cruz do Sul: Editora e Livraria da FISC. 188 p.

Putzke J. \& Putzke M.T.L. (2017) O gênero Agaricus L. ex Fr. (Agaricales, Basidiomycota) no Brasil. Neotropical Biology and Conservation, 12: 109-130.

Putzke J., Putzke M.T.L. \& Köhler A. (2014) Notas sobre os fungos Agaricaceae (Agaricales Basidiomycota) comestíveis encontrados em área em regeneração natural em Santa Cruz do Sul - RS, Brasil. Caderno de Pesquisa, Série Biologia, 25: 44-53.

Raithelhuber J. (1987a) Die Gattung Macrolepiota in Südamerika. Metrodiana, 15: 59-71.

Raithelhuber J. (1987b) Die Gattung Leucocoprinus in ABC-Staden. Metrodiana, 15: 35-44.

Raithelhuber J. (1988) Typenstudien an exsikkaten aus südamerikanischen herbarien. Metrodiana, 1: 5-29.

Redhead S.A. (1987) The Xerulaceae (Basidiomycetes), a family with sarcodimic tissues. Canadian Journal of Botany, 65: 1551-1562.

Redhead S.A., Ammirati J.F., Walker G.R., Norvell L.L. \& Puccio M.B. (1994) Squamanita contortipes, the Rosetta Stone of a mycoparasitic agaric genus. Canadian Journal of Botany, 75: 1812-1824. DOI: 10.1139/b94-223

Redhead S.A., Vilgalys R., Moncalvo J.-M., Johnson J. \& Hopple Jr. J.S. (2001) Coprinus Pers and the disposition of Coprinus species sensu lato. Taxon, 50: 203-241. DOI: 10.2307/1224525

Reijnders A.F. (2000) A morphogenetic analysis of the basic characters of the gasteromycetes and their relation to other basidiomycetes. Mycological Research, 104: 900-910. DOI: 10.1017/S0953756299002233

Rick J. (1905) Pilze aus Rio Grande do Sul. Annales Mycologici, 3: 235-240.

Rick J. (1906) Pilze aus Rio Grande do Sul. Brotéria Série Botânica, 5: 5-53.

Rick J. (1907) Contribution ad monographiam Agaricacinarum Brasiliensium. Brotéria Série Botânica, 6: 65-92.

Rick J. (1908) Fungi Austro-Americani IX-X. Annales Mycologici, 6: 105-108.

Rick J. (1920) Contributio III ad Monographiam Agaricacinarum Brasiliensium. Brotéria Série Botânica, 18: 48-63.

Rick J. (1926) Descrição de algumas espécies novas da micoflora Rio Grandense. Egatea, 11: 1617.

Rick J. (1930) Contributio IV ad monographiam Agaricacinarum Brasiliensium. Brotéria Série Botânica, 24: 27-118.

Rick J. (1937) Agarici Riograndensis. Lilloa, 1: 307-346. 
Rick J. (1938) Agarici Riograndenses. Lilloa, 2: 251-316.

Rick J. (1939) Agarici Riograndensis. Lilloa, 4: 75-104.

Rick J. (1961) Basidiomycetes Eubasidii in Rio Grande do Sul - Brasilia. 5. Iheringia, 8: 296-450.

Rosa L.H., Machado K.M.G., Jacob C.C., Capelari M., Rosa C.A. \& Zani C.L. (2003) Screening of Brazilian Basidiomycetes for antimicrobial activity. Memórias do Instituto Oswaldo Cruz, 98: 967-674. DOI: 10.1590/S0074-02762003000700019

Rother M.S. \& Silveira R.M.B. (2008) Família Agaricaceae (Agarcicales, Basidiomycota) no Parque Estadual de Itapuã, Viamão, Rio Grande do Sul, Brasil. Revista Brasileira de Biociências, 6: 259-268.

Rother M.S. \& Silveira R.M.B. (2009a) Leucoagaricus lilaceus (Agaricaceae), a poorly known Neotropical agaric. Mycotaxon, 107: 473-481. DOI: 10.5248/107.473

Rother M.S. \& Silveira R.M.B. (2009b) Leucocoprinus Pat. (Agaricaceae, Basidiomycota) no Parque Estadual de Itapoã, Viamão, RS, Brasil. Acta Botanica Brasilica, 23: 720-728. DOI: 10.1590/S0102-33062009000300011

Routien J.B. (1940) Two new Gasteromycetes. Mycologia, 32: 159-169.

Saccardo P.A. (1887) Sylloge Fungorum.V. Patavii. 1146 p.

Saville D.B.O. (1968) Possible interrelationships between fungal groups (p. 649-675). In: Ainsworth G.C. \& Sussman A.S. (Eds.). The Fungi: An Advanced Treatise. III. New York: Academic Press. 738 p.

Singer R. (1936) Das System der Agaricales I. Annales Mycologici, 34: 286-378.

Singer R. (1949) The Agaricales (Mushrooms) in Modern Taxonomy. Lilloa, 22: 5-830.

Singer R. (1950) Type studies on Basidiomycetes. IV. Lilloa, 23: 147-246.

Singer R. (1953) Type studies on Basidiomycetes VI. Lilloa, 26: 57-159.

Singer R. (1955) Type studies in Basidiomycetes VIII. Sydowia, 9: 367-431.

Singer R. (1958) The meaning of the affinity of the Secotiaceae with the Agaricales. Sydowia, 12: $1-43$.

Singer R. (1962) The Agaricales in Modern Taxonomy. $2^{\circ}$ edition. Vaduz: J. Cramer.

Singer R. (1973) Diagnoses fungorum novorum Agaricalium III. Beiheft zur Sydowia, 7: 1-106.

Singer R. (1976) Amparoinaceae and Montagneaceae. Revue de Mycologie, 40: 57-64.

Singer R. (1986) The Agaricales in Modern Taxonomy. $4^{\circ}$ edition. Koegnistein: Koeltz Scientific Books. $981 \mathrm{p}$.

Singer R. (1989) New taxa and new combinations of Agaricales (Diagnoses Novorum Agaricalium IV). Fieldiana, Botanica, 21: 1-133.

Singer R. \& Smith A.H. (1960) Studies on secotiaceous fungi. VII. Secotium and Neosecotium. Madroño, 15: 152-158.

Smith S.E. \& Read D.J. (2008) Mycorrhizal Symbiosis. $3^{\circ}$ edition. London: Academic Press. 787 p.

Spegazzini C. (1889) Fungi Puigariani. Boletín de la Academia Nacional de Ciencias en Córdoba, 11: 381-414.

Spielmann A.A. \& Putzke J. (1998) Leucoagaricus gongylophorus (Agaricales, Basidiomycota) em ninho ativo de formigas Attini (Acromyrmex asperus). Caderno Pesquisas Série Botânica, 10: 27-36.

Theissen F. (1912) Hymenomycetes Riograndenses. Brotéria, 10: 1-24.

Thiers H.D. (1984) The secotioid syndrome. Mycologia, 76: 1-8.

Trappe J.M. (1962) Fungus associates of ectotrophic mycorrhizae. The Botanical Review, 28: 538606.

Vellinga E.C. (2000) Notulae ad floram agaricinam Neerlandicam. XXXVIII. Leucoagaricus subgenus Sericeomyces. Persoonia, 17: 473-480.

Vellinga E.C. (2003) Chlorophyllum and Macrolepiota (Agaricaceae) in Australia. Australian Systematic of Botany, 16: 361-370.

Vellinga E.C. (2004) Genera in the family Agaricaceae: evidence from nrITS and nrLSU sequences. Mycological Research, 10: 352-377. DOI: 10.1017/S0953756204009700

Vellinga E.C., de Kok R.P.J. \& Bruns T.D. (2003) Phylogeny and taxonomy of Macrolepiota (Agaricaceae). Mycologia, 95: 442-456. DOI: 10.1080/15572536.2004.11833089 
Vellinga E.C. (2003) Chlorophyllum and Macrolepiota (Agaricaceae) in Australia. Australian Systematic of Botany, 16: 361-370.

Vellinga E.C. \& Yang Z.-L. (2003) Volvolepiota and Macrolepiota - Macrolepiota velosa, a new species from China. Mycotaxon, 85: 183-186.

Vellinga E.C., Sysouphanthong P. \& Hyde K.D. (2011) The family Agaricaceae: phylogenies and two new white-spored genera. Mycologia, 103: 494-509. DOI: 10.3852/10-204

Wartchow F., Putzke J. \& Cavalcanti M.A.Q. (2008) Agaricaceae Fr. (Agaricales, Basidiomycota) from areas of Atlantic Forest in Pernambuco, Brazil. Acta Botanica Brasilica, 22: 287-299. DOI: $10.1590 /$ S0102-33062008000100026

Yokohama K. \& Yamaji D. (1981) Poisoning by Lepiota neomastoidea. Transactions of the Mycological Society of Japan, 22: 255-258.

Zeller S.M. (1943) North American species of Galleropsis, Gyrophragmiun, Longia e Montagnea. Mycologia, 35: 409-417.

Zeller S.M. (1945) A new name. Mycologia, 37(5): 636.

Zeller S.M. (1947) More notes on Gasteromycetes. Mycologia, 39(3): 282-312.

Zeller S.M. (1948) Notes on certain Gasteromycetes, including two new orders. Mycologia, 40: 639-668.

Zeller S.M. \& Walker L.B. (1935) Gasterella, a new uniloculate Gasteromycete. Mycologia, 27: 573-579. 